\title{
Image-guided Pro-angiogenic Therapy in Diabetic Stroke Mouse Models Using a Multi-modal Nanoprobe
}

\author{
Ying-Ying Bai ${ }^{1}$, Xihui Gao ${ }^{2}$, Yuan-Cheng Wang ${ }^{1}$, Xin-Gui Peng ${ }^{1}$, Di Chang ${ }^{1}$, Shuyan Zheng ${ }^{2}$, Cong Li2 ${ }^{\bowtie}$, \\ and Shenghong Ju${ }^{1 凶}$ \\ 1. Jiangsu Key Laboratory of Molecular and Functional Imaging, Department of Radiology, Zhongda Hospital, Medical School, Southeast \\ University, Nanjing, 210009, China; \\ 2. Key Laboratory of Smart Drug Delivery, Ministry of Education \& PLA, School of Pharmacy, Fudan University, Shanghai, 201203, China.
}

$\square$ Corresponding author: Shenghong Ju, jsh0836@hotmail.com; Cong Li, congli@fudan.edu.cn.

() Ivyspring International Publisher. This is an open-access article distributed under the terms of the Creative Commons License (http://creativecommons.org/ licenses/by-nc-nd/3.0/). Reproduction is permitted for personal, noncommercial use, provided that the article is in whole, unmodified, and properly cited.

Received: 2014.04.28; Accepted: 2014.04.29; Published: 2014.05.25

\begin{abstract}
Purpose: The efficacy of pro-angiogenic therapy is difficult to evaluate with current diagnostic modalities. The objectives were to develop a non-invasive imaging strategy to define the temporal characteristics of angiogenesis and to evaluate the response to pro-angiogenic therapy in diabetic stroke mouse models.

Methods: A home-made $\alpha_{v} \beta_{3}$ integrin-targeted multi-modal nanoprobe was intravenously injected into mouse models at set time points after photothrombotic stroke. Magnetic resonance imaging (MRI) and near-infrared fluorescence (NIRF) imaging were carried out at $24 \mathrm{~h}$ post-injection. Bone marrow-derived endothelial progenitor cells (EPCs) were infused into the mouse models of ischemic stroke to stimulate angiogenesis.

Results: The peak signal intensity in the ischemic-angiogenic area of diabetic and wild-type mouse models was achieved on day 10, with significantly lower signal enhancement observed in the diabetic models. Although the signal intensity was significantly higher after EPC treatment in both models, the enhancement was less pronounced in the diabetic animals compared with the wild-type controls. Histological analysis revealed that the microvessel density and expression of $\beta_{3}$ integrin were correlated with the signal intensity assessed with MRI and NIRF imaging.

Conclusions: The non-invasive imaging method could be used for early and accurate evaluation of the response to pro-angiogenic therapy in diabetic stroke models.
\end{abstract}

Key words: angiogenesis, diabetes mellitus, endothelial progenitor cells, molecular imaging, nanoparticle, ischemic stroke.

\section{Introduction}

Stroke is the second leading cause of death globally, and diabetes mellitus is one of the independent risk factors for stroke [1]. Diabetic patients have an increased risk of stroke and exacerbated ischemic cerebral damage and poorer outcomes after stroke [2]. Decreases in the expression of eNOS, angiostatin and vascular endothelial growth factor $[3,4]$ are potential mechanisms underlying impaired angiogenesis after diabetic stroke and lead to an increased infarct volume and poorer outcomes.
The stimulation of angiogenesis can promote endogenous recovery mechanisms in the ischemic brain. Numerous treatments for ischemic stroke have expedited and augmented collateral vessels using various agents, such as statins, nitric oxide agents, and growth factors $[5,6]$. Endothelial progenitor cells (EPCs) could greatly enhance angiogenesis in ischemic stroke models not only through differentiation into endothelial cells in neovessels but also by augmentation of endogenous cytokines, growth factors, 
and chemokines [7-9]. Despite the initial success of EPC transplantation, there has only been one pilot randomized controlled clinical trial of autologous EPC transplantation [10]. The lack of a non-invasive strategy for monitoring therapeutic efficacy is a major limiting factor of clinical trials.

Current clinical techniques used to assess therapeutic efficacy in patients with ischemia, such as angiography and blood flow, could be valuable when alterations occur in large arteries, which form during the later stages of revascularization [11]. An imaging method that is sensitive to revascularization in its early stages could avoid missing the best treatment opportunities and greatly increase the benefit-to-risk ratio for candidate patients who exhibit individual differences. Molecular imaging provides a unique tool for detecting angiogenesis [12]. Among all available imaging technologies, magnetic resonance imaging (MRI) does not involve ionizing radiation, offers high spatial resolution, and provides the ability to visualize tissues without limitations of depth and angle. On the other hand, near-infrared fluorescence (NIRF) imaging is easily operated and very sensitive. Therefore, a dual-modal strategy that combines MRI and NIRF imaging will achieve both high spatial resolution and sensitivity.

The $a_{v} \beta_{3}$ integrin, which is up-regulated in neovascular endothelial cells, is ideal for the detection of angiogenesis [13]. We have previously demonstrated that $\alpha_{v} \beta_{3}$ integrin is a reliable angiogenesis marker for detecting brain tumors. A peptide in our targeted nanoprobes containing a cyclic arginine-glycine-aspartic acid (cRGD) sequence has shown a high affinity for $\alpha_{v} \beta_{3}$ integrin [14]. Although $\alpha_{v} \beta_{3}$ integrin was expressed in microvessels after cerebral ischemia [15], there are few studies using cRGD-conjugated nanoprobes to detect the pro-angiogenic response to ischemic stroke, particularly in diabetic animal models.

Therefore, the overall objectives of this study were to explore a non-invasive strategy to evaluate angiogenesis in ischemic stroke models and to compare the angiogenesis patterns between $d b / d b$ and wild-type (WT) mice after stroke, as well as to monitor their responses to pro-angiogenic therapy.

\section{Methods and Materials}

\section{Synthesis of the Nanoprobes}

The multi-modal nanoprobes were synthesized as previously reported [14]. The cyclic peptide cRGDyK (GL Biochem, China), Gd ${ }^{3+}$-DTPA, IR783 (home-made [16]), and rhodamine (Sigma-Aldrich, USA) were functionalized into the fifth generation (G5) PAMAM dendrimer (Weihai CY Dendrimer Technology, China) to generate the $\alpha_{v} \beta_{3}$ integ- rin-targeted nanoprobe Den-RGD. The control nanoprobe Den-PEG, which has a similar chemical structure to Den-RGD but without the cyclic peptide-targeting domain, was also prepared. The characterization of the nanoprobes is described in the supplementary material.

\section{Cerebral Ischemic Stroke Model}

All animal experiments were approved by the Institutional Animal Use and Care Committee of the Medical School of Southeast University. Cerebral ischemic stroke was induced via photothrombosis in adult C57BL/ 6 and $d b / d b$ mice (male, 8 weeks old, Academy of Military Medical Science, China). The $d b / d b$ (leptin-receptor-deficient) mouse is a recognized model of type 2 diabetes, with blood glucose levels that ranged from 14.6-29.9 mmol/L in this study. The mice were anesthetized with $1 \%$ isoflurane (KeYuan, China) using a gas anesthesia mask. Rose bengal (100 $\mathrm{mg} / \mathrm{kg}$, Sigma-Aldrich, USA) was injected intraperitoneally 5 min prior to illumination. For illumination, a 4-mm-diameter fiber of a cold light source (Zeiss, Germany) was centered $2 \mathrm{~mm}$ to the right of the bregma after hair was removed [17]. The brains were illuminated for $15 \mathrm{~min}$, and the mice then recovered from anesthesia. Photothrombotic ischemia was verified via $\mathrm{T}_{2}$-weighted imaging $24 \mathrm{~h}$ after surgery.

\section{Culture, Characterization, and Transplantation of EPCs}

EPCs were generated from mononuclear cells of C57BL/ 6 mice (male, 5 weeks old) as we previously reported [18]. The mononuclear cell fraction from the tibias and femurs was collected via ficoll density gradient centrifugation. The cells were then suspended in Endothelial Basal Media-2 (EBM-2, Lonza, Basel, Switzerland) supplemented with growth factors. The characterization of EPCs is described in the supplementary material.

After 14 days of culture, $100 \mu \mathrm{L}$ of the cell suspension $\left(1 \times 10^{6}\right.$ cells) or saline was randomly pumped into mice $24 \mathrm{~h}$ post ischemia via the ipsilateral internal carotid artery through a PE catheter (AniLab, China).

\section{Experimental Groups}

The in vivo experiment consisted of three studies. The mouse models without an ischemic lesion on $\mathrm{T}_{2} \mathrm{WI}(2 d b / d b$ mice) or dead mice $(4 d b / d b$ mice died within $24 \mathrm{~h}$ of photothrombotic stroke) were excluded from the experiment.

STUDY 1. To demonstrate the targeting specificity of angiogenesis in vivo, WT cerebral ischemic stroke mice were randomly assigned to 1 of 4 groups on day 7 and administered the following treatments: (1) $\alpha_{v} \beta_{3}$ integrin-targeted nanoprobe Den-RGD (290 $\mathrm{nmol} / \mathrm{kg}, \mathrm{n}=9$ ); (2) Control nanoprobe Den-PEG (290 
$\mathrm{nmol} / \mathrm{kg}, \mathrm{n}=9)$; (3) Den-RGD (290 nmol/kg) with free cRGDyk peptide $(29 \mu \mathrm{mol} / \mathrm{kg})$ pretreatment $(\mathrm{n}=6)$; or (4) Saline ( $n=6)$.

STUDY 2. To dynamically evaluate angiogenesis after stroke, the diabetic and WT mouse models were randomly assigned to different time points (days $3,7,10,14$ and $21 ; \mathrm{n}=10$ or 12 , respectively).

STUDY 3. For pro-angiogenic therapy, the diabetic and WT mouse models were randomly assigned to receive an EPC infusion or saline infusion on day 1 ( $\mathrm{n}=24$ per group).

\section{Magnetic Resonance Imaging}

MRI was performed using a 7.0-Tesla small animal MR scanner (Bruker PharmaScan, Germany). For cell phantom imaging, $2 \times 10^{6}$ cells were suspended in $1 \mathrm{~mL}$ of $1 \%$ agarose (Oxold, UK) in 10-mm-diameter Eppendorf tubes (Corning Incorporated, USA). The mice were anesthetized via a continuous supplying of $1 \%$ isoflurane, and their respiratory rate and body temperature were monitored via a physiology monitor.

Spin echo sequence $(500 / 15$ msec of repetition time/echo time, 8 averages) was used for $\mathrm{T}_{1}$-weighted imaging and fast spin echo sequence $(2,000 / 50 \mathrm{msec}$ of repetition time/echo time, 1 average) was used for $\mathrm{T}_{2}$-weighted imaging. Twelve axial slices with a slice thickness of $1 \mathrm{~mm}$, matrix of $256 \times 256$, and field of view of $2 \times 2 \mathrm{~cm}$ were positioned over the brain. Region of interest (ROI) was drawn in the peri-infarct area on the $\mathrm{T}_{1}$-weighted images. The contrast-to-noise ratio $(\mathrm{CNR})$ was defined as: $\mathrm{CNR}=\left(\mathrm{SI}_{\mathrm{P}}-\mathrm{SI}_{\mathrm{M}}\right) / \mathrm{SI}_{\mathrm{N}}$, in which $\mathrm{SI}_{\mathrm{P}}=$ signal intensity of the peri-infarct area, $\mathrm{SI}_{\mathrm{M}}$ = signal intensity of the temporalis, and $\mathrm{SI}_{\mathrm{N}}=$ signal intensity of the background noise. Relative CNR was defined as follows: (CNR of the images collected $24 \mathrm{~h}$ after the nanoprobe injection)/(CNR of the corresponding images collected prior to the injection).

\section{Near-infrared Fluorescence Imaging}

MRI scanning was followed by NIRF imaging using the Maestro In vivo Imaging System (CRi, USA). After the mice were anesthetized and monitored as described for the MRI scanning, the skull of each mouse was exposed via a 20-mm-long skin incision prior to in vivo imaging. The NIRF images were captured at an excitation wavelength of $745 \mathrm{~nm}$ and an emission wavelength of $783 \mathrm{~nm}$ with an exposure time of $2 \mathrm{~s}$. After the mice were euthanized, the brain, heart, spleen, liver, kidneys, and lungs were surgically dissected for ex vivo imaging. The parameters were the same for ex vivo imaging and for in vivo imaging.

The pure IR783-related fluorescence signal in the NIRF images was extracted from the autofluorescence signal based on their spectral patterns using multi- spectral imaging capabilities implemented in Maestro 2.10.0 software. The NIRF signal intensity from the explanted organs was quantified as the counts per second per pixel by CRi Maestro software. For the analysis of signal changes at a series of time points and in different groups, rectangular ROIs were manually drawn in the regions of the right and left hemispheres on the ex vivo images. The target-to-background ratio (TBR) was calculated as follows: (ROI value from the right hemisphere)/(ROI value from the left hemisphere) [19].

\section{Behavioral Tests}

Behavioral tests were performed in the mouse models of STUDY 3. The assessments included the modified neurological severity score (mNSS) and the foot-fault test $[20,21]$; the test-related details are described in the supplementary material.

\section{Immunohistochemical Staining}

Consecutive sections of the brains with a thickness of $6 \mu \mathrm{m}$ were sliced in a freezing microtome (Leica CM 1950, Germany). To confirm the presence of Den-RGD in the neovasculature, the brain sections were incubated with a rabbit anti-mouse $\beta_{3}$ integrin monoclonal antibody (1:50 dilution, Abcam, China), which was followed by staining with a goat anti-rabbit Alexa Fluor 488 polyclonal antibody (1:500 dilution, Life Technologies, USA). Nuclear DNA was stained with DAPI (1:200 dilution, Sigma-Aldrich, USA).

The sections stained with CD31 antibody (1:50 dilution, Abcam, China) were used for measuring the microvessel density (MVD). The CD31-positive cells in the peri-infarct region were counted from 6 mice per group in a double-blind manner [7].

\section{Quantification of Gadolinium in Tissues}

The nanoprobes present in the tissues were quantified based on the gadolinium contents. Fresh brain hemispheres with ischemia obtained from different groups of mice at different time points were weighed (approximately $100 \mathrm{mg}$ ) and digested as we previously described [22]. The quantification was measured via inductively coupled plasma mass spectrometry (ICP-MS, PerkinElmer, USA).

\section{Western Blot Analysis}

Brain hemispheres with ischemia obtained from different groups of mice were frozen and stored at $-70^{\circ} \mathrm{C}$ after dissection. Protein concentrations were calculated by the bicinchoninic acid method. The protein was subjected to SDS-PAGE and then transferred to polyvinylidenefluoride membranes (Millipore, USA). Blots were incubated with rabbit anti-mouse $\beta_{3}$ integrin and GAPDH antibodies (Abcam, 
China) at $4^{\circ} \mathrm{C}$ overnight after blocking with $5 \%$ skim milk (BD Bioscience, USA). Binding was detected with horseradish peroxidase conjugated secondary antibody (Life Technologies, USA). The proteins levels (band densities) were quantified using ImageJ software (NIH, USA).

\section{Statistical Analysis}

Numerical data are presented as the mean \pm standard deviation (SD). Least significant difference tests after one-way ANOVA were used to compare the CNR, TBR, Gd contents, expression of $\beta_{3}$ integrin, behavioral tests, and MVD obtained from four groups in the cell experiments, STUDY 1 , and STUDY 3 . The data (CNR, TBR, Gd contents, and expression of $\beta_{3}$ integrin) obtained from the diabetic and WT mice at different time points in STUDY 2 were analyzed with Student's $t$ test. Correlations between in vivo imaging and the expression of $\beta_{3}$ integrin or MVD in the histological analysis were analyzed by Pearson's correlation coefficients. $\mathrm{P}<0.05$ was considered statistically significant.

\section{Results}

\section{Synthesis and Characterization of the Nanoprobes}

The chemical structures of the neovasculature-targeting and control nanoprobes are presented in Figure 1. The PAMAM dendrimer was selected as a scaffold because of its optimized circulation lifetime and identical molecular weight. The NIR fluorophore IR783 was used to label the dendrimer because of its high extinction coefficient and quantum yield. Conjugated rhodamine was employed to track the nanoprobes in histology. $\mathrm{Gd}^{3+}$-DTPA was chosen as a paramagnetic chelator functionalized on the nanoprobe due to its convenience regarding conjugation with the nanoparticle.

The hydrodynamic diameter of the targeted nanoprobe Den-RGD was $15.81 \mathrm{~nm}$, which was slightly larger than the $11.55 \mathrm{~nm}$ of the Den-PEG control nanoprobe. The molar ratios of PAMAM:PEG:RGD: DTPA were measured as 1:8.2:6.9:60. The nanoprobes contained $46.3 \pm 1.6 \mathrm{Gd}^{3+}$ atoms per particle and demonstrated similar longitudinal relaxivity $\mathrm{r}_{1 \mathrm{p}}$ values of $2.4 \pm 0.7 \mathrm{mM}^{-1} \mathrm{~s}^{-1} / \mathrm{Gd}^{3+}$.

\section{Cytotoxicity of Nanoprobes and Cellular Uptake Studies}

The cytotoxicity and cellular uptake of the nanoprobes are described in the supplementary material. Compared with the control group treated with phosphate-buffered saline (PBS), MTT absorbance values of the cells incubated with different concentrations of nanoprobes $(2.5,5,10$, and $20 \mu \mathrm{M})$ showed no significant cell cytotoxicity for either nanoprobe (Supplementary Material: Figure S1A).

The binding specificity of Den-RGD was first demonstrated in the $\alpha_{v} \beta_{3}$ integrin-positive U87MG cells. The signal intensities of the cells on MR and NIRF images were significantly higher in the Den-RGD group compared with the Den-PEG group (Supplementary Material: Figure S1B to S1D). The majority of the signal intensity in the Den-RGD group was blocked by pre-treatment with free c(RGDyk) (Supplementary Material: Figure S1B to S1D). In vitro immunofluorescence staining also confirmed that the Den-RGD was attached to the cell membrane (Supplementary Material: Figure S2). Den-RGD demonstrated greater cellular uptake than the control nanoprobe.

\section{Targeting Angiogenesis in Cerebral Ischemic Stroke Mice}

The $\alpha_{v} \beta_{3}$ integrin-targeting ability of Den-RGD was further investigated using in vivo $\mathrm{T}_{1}$-weighted MRI and NIRF imaging of ischemic stroke mice. Compared with the $\mathrm{T}_{1}$-weighted imaging at baseline, a significantly higher CNR appeared in the peri-infarct area at $24 \mathrm{~h}$ after injection of Den-RGD compared with Den-PEG (Figure 2A and 2B). The TBR observed on the NIRF images was significantly higher in the Den-RGD group compared with the Den-PEG group (Figure 2C). The administration of Den-RGD together with the pretreatment of the blocking cRGDyk peptide revealed significantly reduced uptake in the brain (Figure 2B and 2D, n=6).
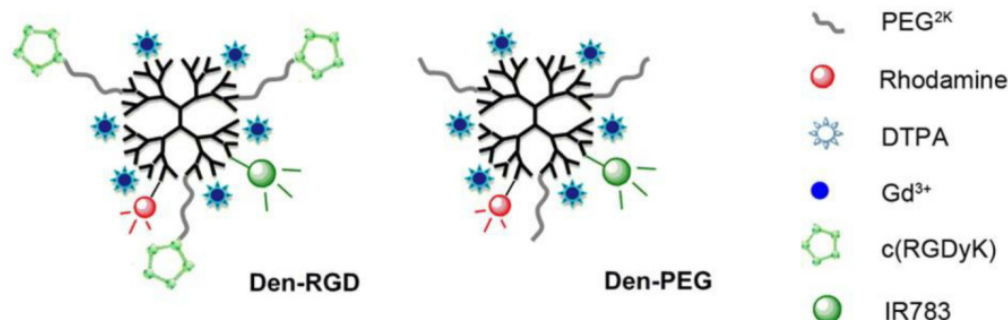

Figure I: Chemical structures of Den-RGD and Den-PEG. The cyclic peptide cRGDyK, Gd ${ }^{3+}$-DTPA, IR783, and rhodamine were functionalized into the fifth generation PAMAM dendrimer to synthesize the $\alpha_{v} \beta_{3}$ integrin-targeted nanoprobe Den-RGD. The control nanoprobe Den-PEG, which had a similar chemical structure to Den-RGD but without the cyclic peptide targeting domain, was prepared. 
A

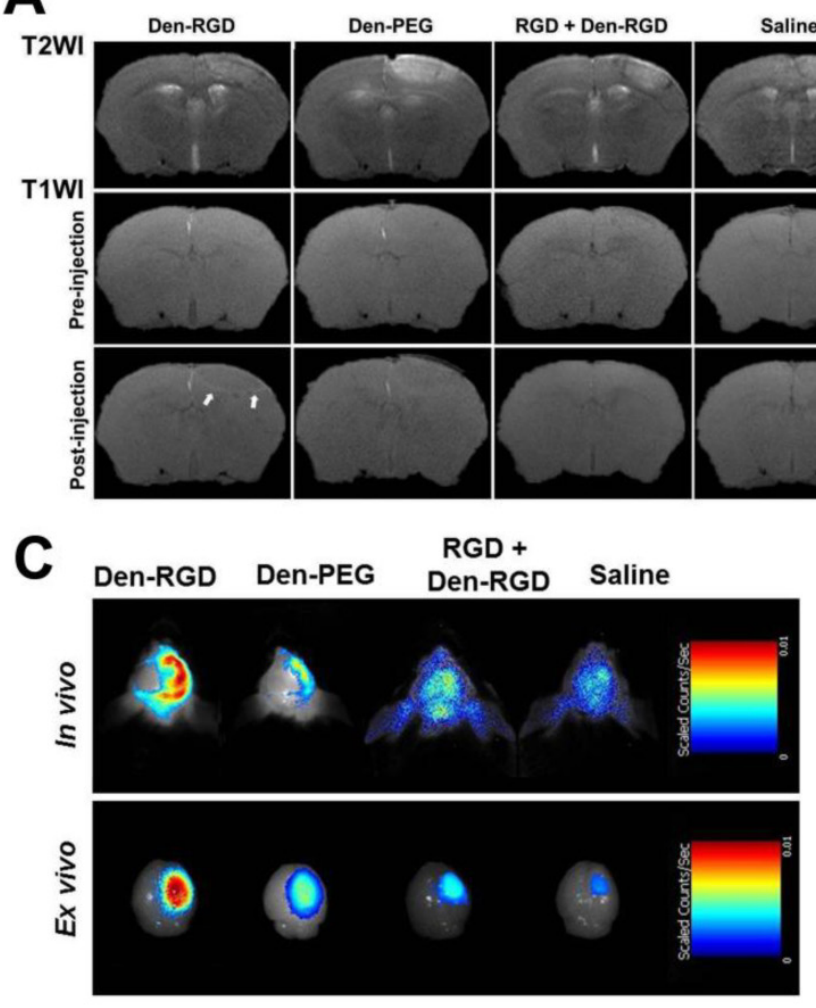

E
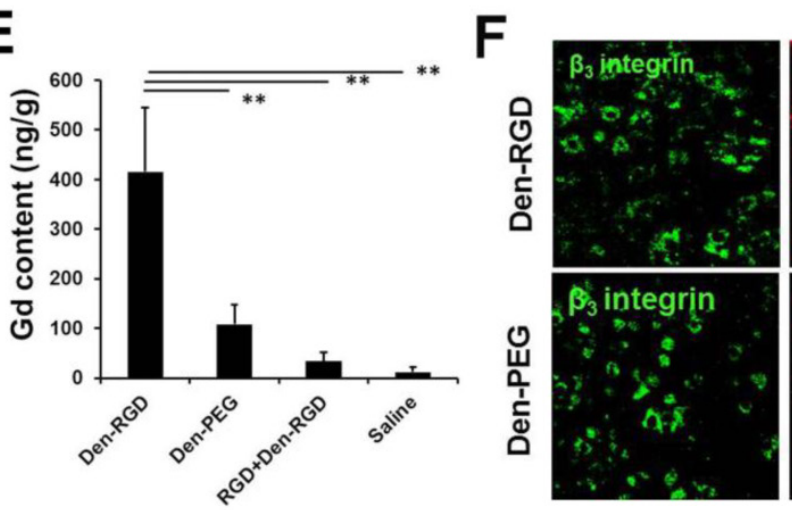

D
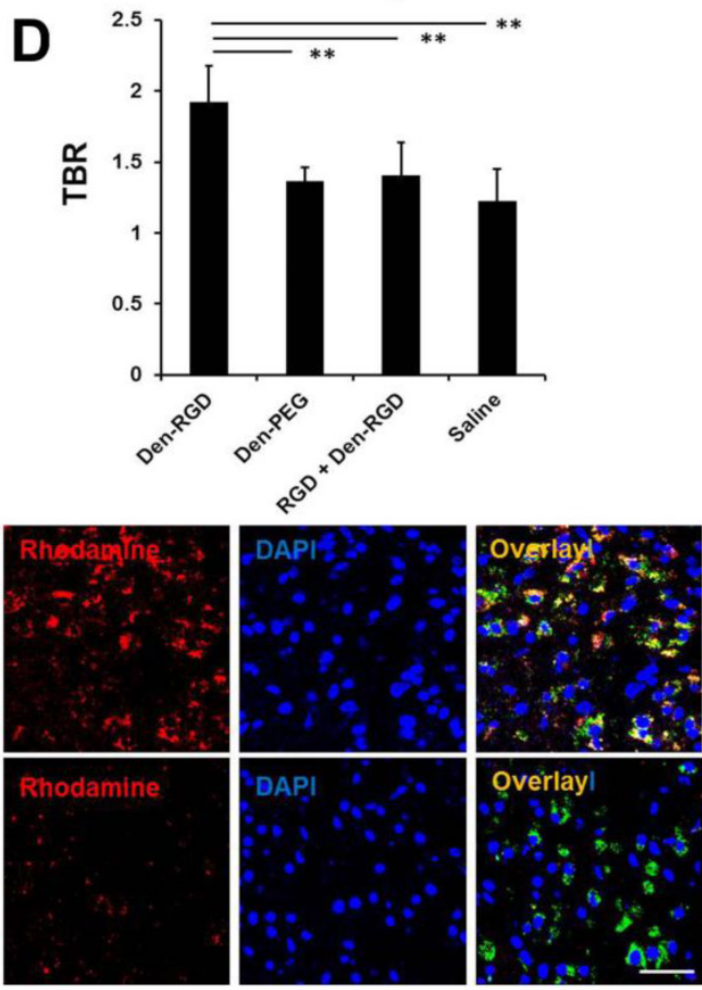

Figure 2: Den-RGD demonstrated a high binding affinity to neovessels in ischemic stroke models. (A) Arrows indicate the high signal on the $T_{1}$-weighted images in the Den-RGD group. The corresponding infract areas are shown on the $\mathrm{T}_{2}$-weighted images. (B) Specific imaging of angiogenesis with Den-RGD produced a 5 I\% higher contrast-to-noise ratio (CNR) in the peri-infarct area on the $T_{1}$-weighted MR images compared with baseline, whereas the non-targeted nanoprobe Den-PEG produced only $14 \%$ CNR enhancement. The signal enhancement caused by Den-RGD was significantly reduced by pre-blocking the integrin receptors with free $c(R G D y k)(n=6)$. (C) Representative NIRF images and (D) quantitative data showed a significantly higher target-to-background ratio (TBR) of the stroke area in the Den-RGD group compared with the other three groups ( $\mathrm{n}=6$ ). (E) $A$ significantly higher gadolinium content in the cerebral hemisphere with ischemia was observed in the Den-RGD group compared with the other groups ( $\mathrm{n}=6$ ). ( $\mathrm{F}$ ) Immunofluorescence staining of brain sections confirmed that the nanoprobe Den-RGD colocalized with the $\beta_{3}$ integrin-positive neovasculature in the peri-infarct area, whereas a minimal amount of the control nanoprobe Den-PEG was observed in the neovasculature. Scale bar $=50 \mu \mathrm{m}, * * \mathrm{p}<0.01$.

After MRI and NIRF imaging, the brain of each mouse was excised for immunofluorescence staining and ICP-MS. A significantly higher gadolinium content in the cerebral hemisphere with ischemia was observed in the Den-RGD group compared with the other groups (Figure 2E, $n=6$ ). Immunofluorescence staining of the brain sections confirmed that the targeted nanoprobe Den-RGD colocalized with the $\beta_{3}$ integrin-positive neovasculature in the peri-infarct area, whereas a minimal level of the control nanoprobe Den-PEG was observed in the neovasculature (Figure 2F).

\section{Nanoprobe Biodistribution Assay}

To study the tissue distribution of the nanoprobes, the heart, liver, spleen, kidneys, and lungs of the mice were surgically removed $24 \mathrm{~h}$ after the delivery of the nanoprobes through the tail vein. The IR783 levels in different organs were quantified through ex vivo NIRF imaging. As illustrated in Supplementary Material: Figure S3A and S3B, the nanoparticles primarily accumulated in the reticuloendothelial system (such as the liver and spleen) and the urinary system (kidneys); these findings are similar to 
the in vivo behaviors of many other nanomaterials applied in biomedicine [23]. Our results showed that clearance of the nanoprobes might occur through both renal and fecal excretion.

\section{Dynamic Evaluation of Angiogenesis after Stroke in Diabetic and WT Mice}

To dynamically evaluate angiogenesis in diabetic and WT mice after ischemic stroke, MRI and NIRF imaging were performed on days 3, 7, 10, 14, and 21 ( $n=5$ or 6 , respectively). A baseline MRI was obtained, and Den-RGD was then injected into the mice $24 \mathrm{~h}$ prior to the set time points. The highest
CNR in the peri-infarct area observed on the $\mathrm{T}_{1}$-weighted images was attained on day 10 after stroke in both animal models, with a significantly lower CNR being observed in the diabetic mice compared with the WT mice at each time point (Figure 3A and 3B). Similarly, the in vivo and ex vivo NIRF images indicated that the TBR reached its peak on day 10 after ischemia in both models, but a significantly lower TBR was observed in the diabetic mice (Figure 3C and 3D). A linear correlation was identified between the CNR on the $\mathrm{T}_{1}$-weighted images and the TBR on NIRF images (Figure 3E, $\mathrm{r}=0.898, \mathrm{p}<0.001$ ).
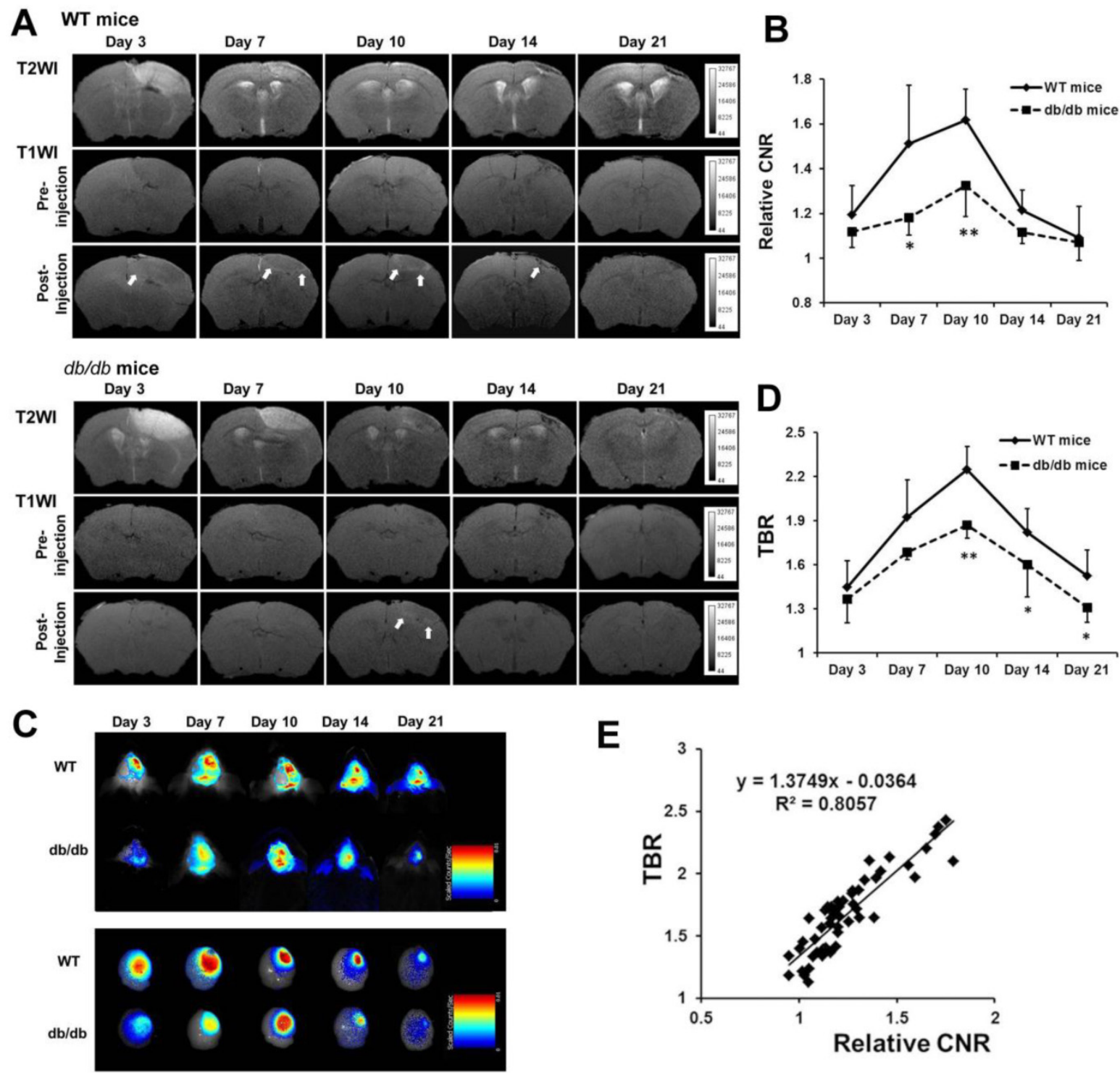

Figure 3: Dynamic evaluation of angiogenesis after stroke in diabetic and WT mice in vivo. Den-RGD was intravenously injected to mice at each time point after surgery. (A) Representative MR images of diabetic and WT mouse brains are shown. (B) The peak of the contrast-to-noise ratio (CNR) on the TI-weighted images occured on day I0 in both groups, with significantly lower signal enhancement in the diabetic mice $(n=5$ or 6$)$. (C) Representative color-coded NIRF images of diabetic and WT mice after the injection of Den-RGD are presented. (D) The target-to-background ratio (TBR) of two animals both peaked on day 10 ( $n=5$ or 6 ). (E) A linear correlation was found between the CNR on the MR images and the TBR on the NIRF images at all the time points $(r=0.898, p<0.001)$. ${ }^{*} p<0.05$, $* * p<0.01$ vs WT mice. 

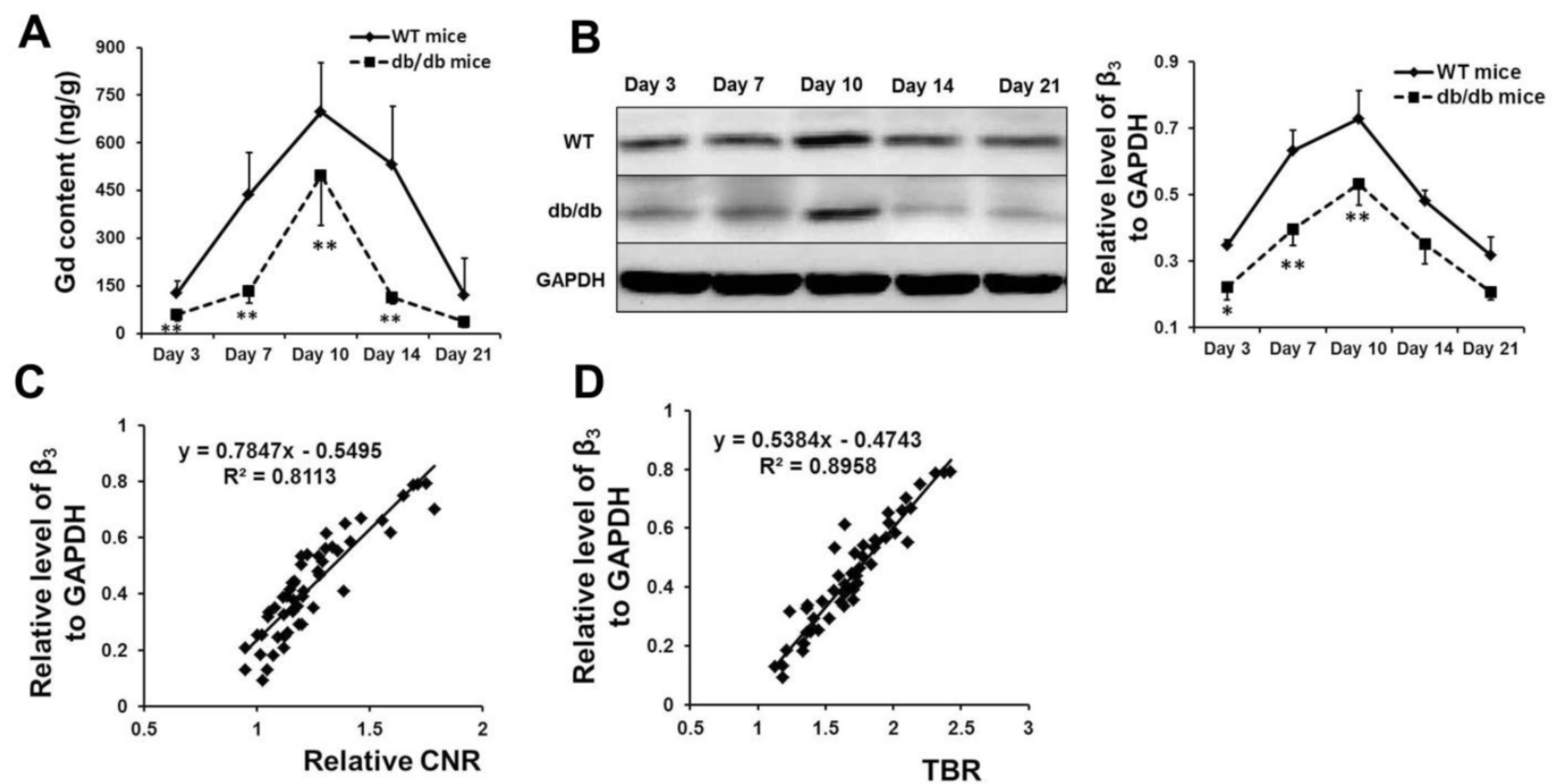

Figure 4: Histological analysis verified the high signal of Den-RGD on the MR and NIRF images. The content of gadolinium (A) and the expression of $\beta_{3}$ integrin (B) in the cerebral hemisphere with ischemia in both models at different time points were detected via ICP-MS and western blot analysis, respectively ( $\mathrm{n}=5$ or 6 ). (C, D) $A$ linear correlation was identified between the expression of $\beta_{3}$ integrin and the contrast-to-noise ratio (CNR) on the MR images $(r=0.90 \mathrm{I}, \mathrm{P}<0.00 \mathrm{I})$ or the target-to-background ratio (TBR) on the NIRF images $(r=0.949, p<0.001)$. ${ }^{*} p<0.05$, ** $p<0.01$ vs WT mice.

The content of gadolinium (Figure $4 \mathrm{~A}$ ) and the expression of $\beta_{3}$ integrin (Figure 4B) in the cerebral hemisphere with ischemia in both models ( $\mathrm{n}=5$ or 6 ) were detected at different time points via ICP-MS and Western blot analyses, respectively. The results verified the findings from MRI and NIRF imaging. A linear correlation was identified between the expression of $\beta_{3}$ integrin and the CNR on the $T_{1}$-weighted images (Figure $4 \mathrm{C}, \mathrm{r}=0.901, \mathrm{p}<0.001$ ) or the TBR on the NIRF images (Figure 4D, $r=0.949, p<0.001$ ).

\section{EPC Characterization}

Bone marrow-derived EPCs were defined as cells that up-take Dil-acetylated low-density lipoprotein (acLDL), bind to FITC-ulex europaeus agglutinin (UEA), and express CD133/CD34/VEGFR2 (Supplementary Material: Figure S4A and S4B). After 14 days of culture, $81.53 \pm 3.61 \%$ of the cells $(n=3)$ expressed CD133, $40.31 \pm 6.02 \%$ expressed CD34, and $44.64 \pm 5.27 \%$ expressed VEGFR-2 (Supplementary Material: Figure S4C).

\section{Responses to Pro-angiogenic Therapy in Diabetic and WT Mouse Models}

The responses to EPC therapy in the diabetic and WT mouse models were examined on day 10 $(\mathrm{n}=6)$ using in vivo imaging systems. The CNR detected by MRI was significantly higher in the WT mice treated with EPCs compared with the mice treated with saline. In the diabetic mice, EPC treatment caused a moderate increase in the CNR on the $\mathrm{T}_{1}$-weighted images, but the increase was weaker compared with the WT mice treated with EPCs (Figure 5A and 5B). Through NIRF imaging, a significant increase of the TBR was detected after EPC treatment in both the WT and diabetic ischemic mouse models (Figure 5C and 5D).

In our ex vivo experiments, the content of gadolinium (Figure $5 \mathrm{E}, \mathrm{n}=6$ ) and the expression of $\beta_{3}$ integrin (Figure $5 \mathrm{~F}, \mathrm{n}=6$ ) in the cerebral hemisphere with ischemia were significantly increased after EPC treatment in both models. The cerebral microvessel density in the two mouse models was significantly higher in the EPC-treated group compared with the saline-treated group (Figure $6 \mathrm{~A}$ and $6 \mathrm{~B}, \mathrm{n}=6$ ), but the number of newly growing capillaries in the WT mice $\left[(51.24 \pm 31.03) / \mathrm{mm}^{2}\right]$ was greater compared with the diabetic mice $\left[(37.11 \pm 14.61) / \mathrm{mm}^{2}\right]$. A linear correlation was detected between the MVD in the histology and the CNR on the MR images (Figure 6C, r $=0.931$, $p<0.001$ ) or the TBR on the NIRF images (Figure 6D, $r$ $=0.890, p<0.001)$. In agreement with the MVD findings, we also observed that the neurological deficit score was reduced in the EPC-treated group in both models on days 14 and $21(n=6)$. The infusion of EPCs improved neurologic motor function in the WT models as early as day 10 and demonstrated a better efficacy on days 14 and 21 compared with the diabetic models (Supplementary Material: Figure S4D). These data confirmed that the in vivo dual-modal imaging 
performed in this work is valid for detecting pro-angiogenic responses during the treatment of brain ischemia.

\section{Discussion}

It is well documented that the neurovasculature in the ischemic penumbra is correlated with the survival rate of stroke patients, and stimulating cerebral angiogenesis has demonstrated great potential for the treatment of ischemic stroke [24]. Although many methods used to stimulate the growth of cerebral vessels in the ischemic brain have been explored [5,6], a non-invasive, clinically feasible and economical method for the evaluation of angiogenic responses to these interventions remains to be developed. This study has demonstrated that non-invasive molecular imaging with multi-modal nanoprobes that target $\mathrm{a}_{\mathrm{v}} \beta_{3}$ integrin can be utilized to detect angiogenesis in cerebral ischemic stroke animal models.
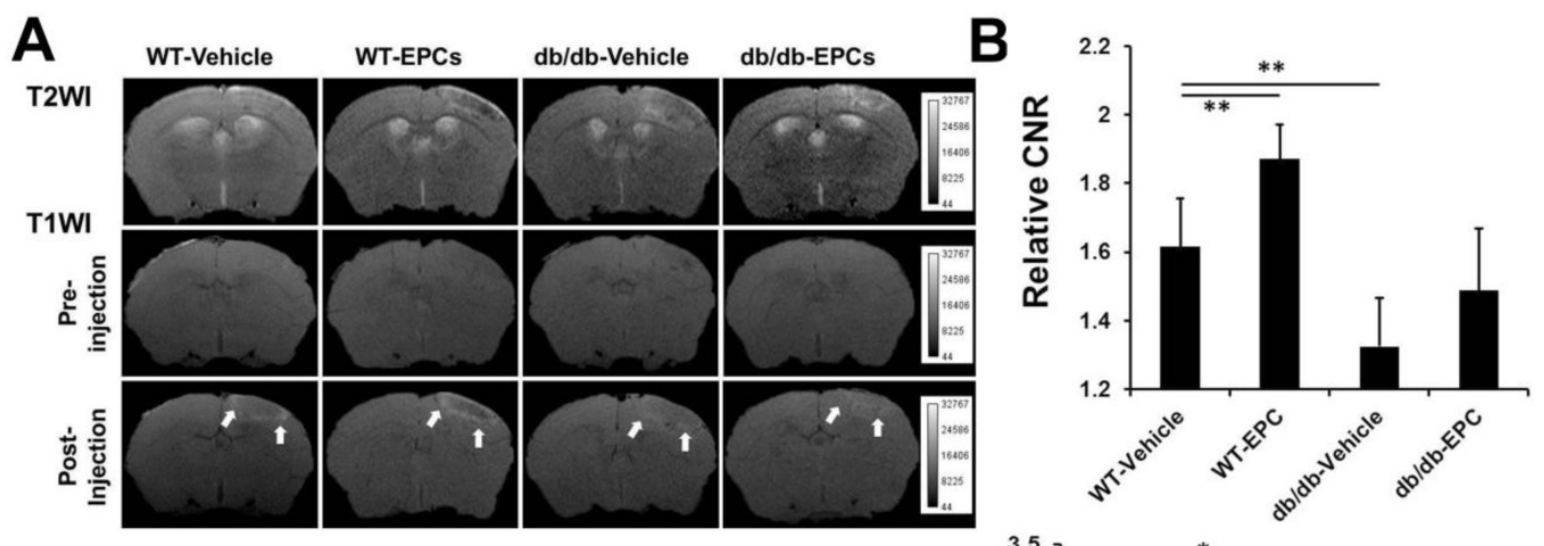

C
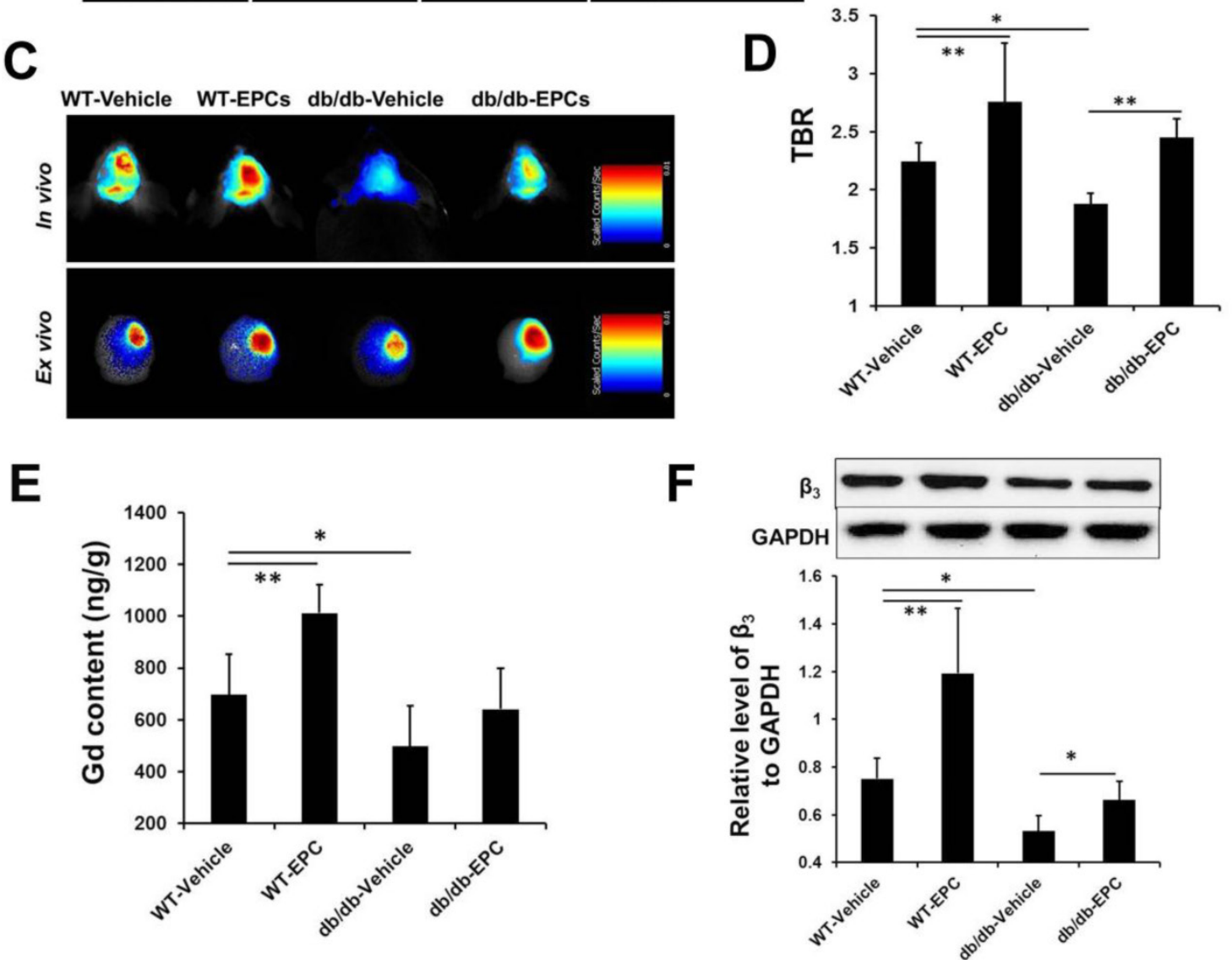

Figure 5: MRI and NIRF imaging and histological analysis of EPC therapy in ischemic stroke mice. (A) Representative MR images of diabetic and WT mice infused with EPC or saline. (B) The contrast-to-noise ratio (CNR) in the WT mice treated with EPC was significantly higher compared with the mice treated with saline. In the diabetic mice, EPC treatment also caused a moderate increase of the CNR on the $T_{1}$-weighted images $(n=6)$. (C, D) With respect to NIRF imaging, EPC therapy was caused a significant increase of the target-to-background ratio (TBR) in both the diabetic and WT mice $(n=6)$. (E) The gadolinium content in the cerebral hemisphere with ischemia was consistent with the signal enhancement detected on the $T_{1}$-weighted images $(n=6)$. $(F)$ The expressions of $\beta_{3}$ integrin in the EPC-treated diabetic and WT mice were significantly higher compared with the saline-treated mice $(n=6)$. $* p<0.05, * * p<0.01$. 


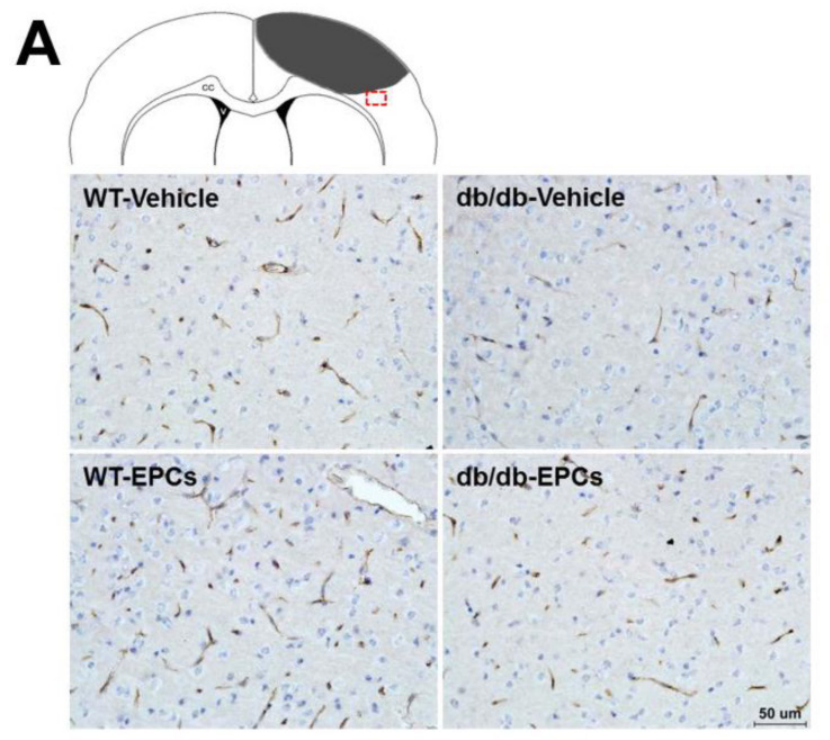

B
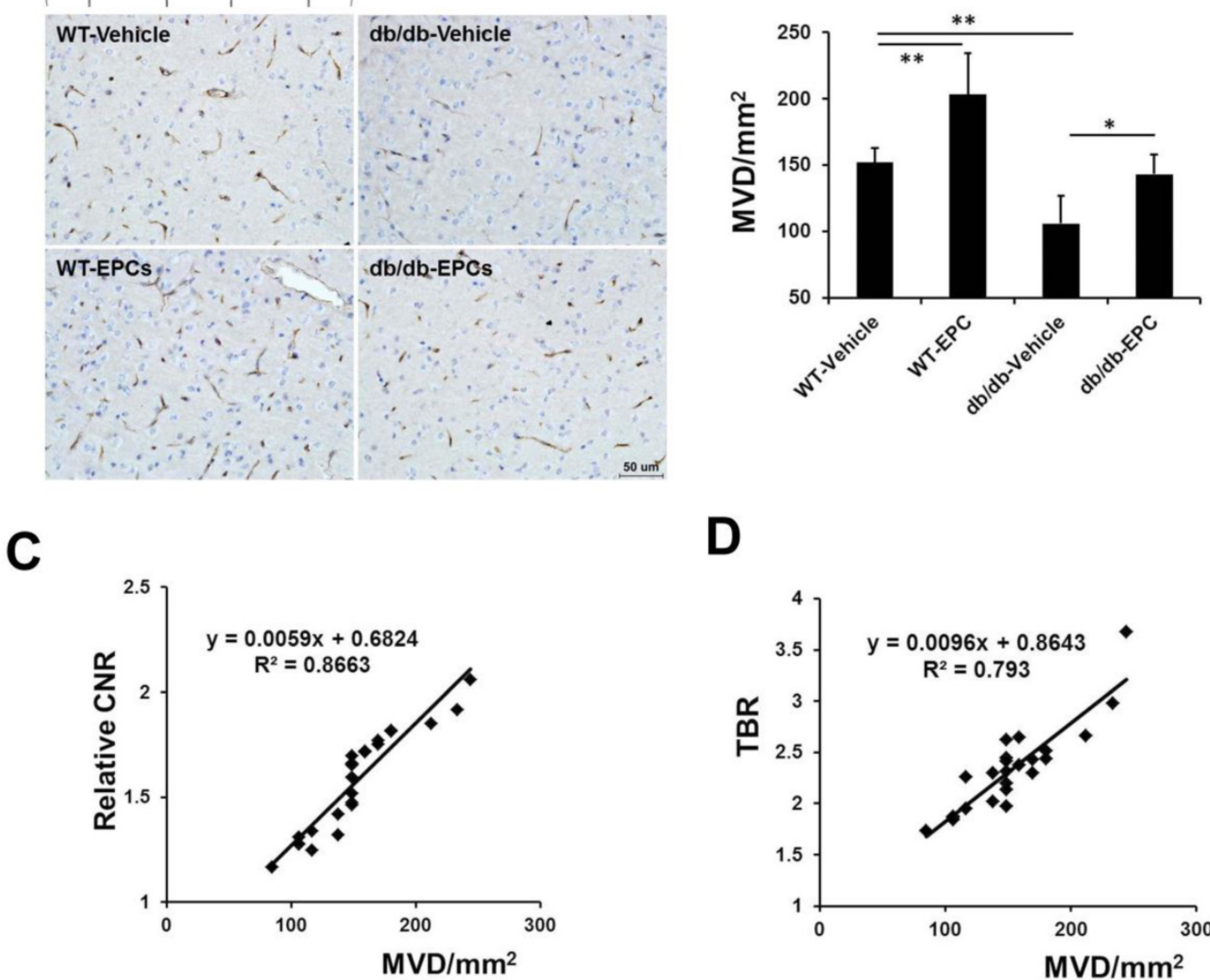

D

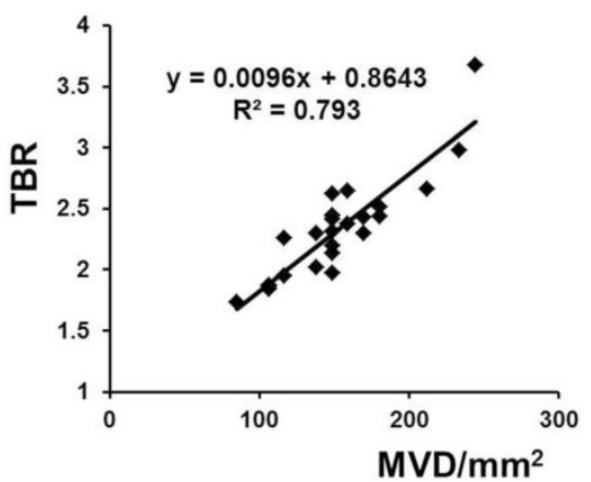

Figure 6: Microvessel density (MVD) in brain samples from the diabetic and WT mice with EPC or saline treatment. (A) Representative images of CD3I staining in the ischemic border zone of mice from different groups. Scale bar $=50 \mu \mathrm{m}$. (B) Staining of the microvasculature showed a greater number of capillaries in the EPC-treated mice compared with the saline-treated mice $(n=6)$. (C, D) A linear correlation was identified between the MVD in the histology and the contrast-to-noise ratio (CNR) on the MR images ( $r=0.93 \mathrm{I}$, $\mathrm{p}<0.00 \mathrm{I})$ or the target-to-background ratio (TBR) on the NIRF images $(r=0.890, p<0.001)$. ${ }^{*} p<0.05, * * p<0.01$.

It has been reported that molecular imaging for monitoring the angiogenesis of tumors with agents that specifically target $\alpha_{v} \beta_{3}$ integrin can be achieved by MRI [25,26], NIRF imaging [27,28], positron-emission tomography (PET) $[28,29]$, and single-photon emission computed tomography (SPECT) $[30,31]$. Although we also used $a_{v} \beta_{3}$ integrin as the target of imaging, pathological angiogenesis in tumors is substantially different from physiological angiogenesis after cerebral ischemia. To date, there has only been one study that utilized a radioactive ${ }^{68} \mathrm{Ga}-\mathrm{RGD}$ probe to evaluate angiogenesis by PET [32]. These radiolabeled peptides have also been used to detect the expression of $\alpha_{v} \beta_{3}$ integrin in other ischemic diseases [33]. However, the spatial resolution of PET or SPECT (mm range) is much lower compared with MRI ( $\mu \mathrm{m}$ range) and cannot provide anatomical information. Additionally, the cost of radionuclide tracers is high, and there is a potential risk of exposure to radioactivity. The dual-modal imaging applied in this study provides a non-invasive method with high resolution, sensitivity, and safety. The targeted multi-modal nanoprobe Den-RGD produced an approximately 3-fold higher signal in the peri-infarct area on MR and NIRF imaging compared with the control nanoprobe Den-PEG. Enhanced blood brain barrier permeability in ischemic territories was reported previously due to disrupted tight junctions between endothelial cells [34]. Just like enhanced permeability and retention effect that was widely used to up-regulate the intra-tumoral uptake of the nanoparticles, the accumulation of Den-PEG, which has a hydrodynamic diameter of $11.55 \mathrm{~nm}$, in the ischemic region can be explained by its prolonged circulation lifetime and the leaky vasculatures present in ischemic brain tissues. The specificity of Den-RGD in the neovasculature was also demonstrated in a blocking assay that utilized a competitive peptide. Ex vivo experiments have further demonstrated the co-localization of Den-RGD with $\beta_{3}$ integrin. These 
data confirmed that Den-RGD is a specific dual-modal imaging-compatible nanoprobe for angiogenesis in cerebral ischemia.

An analysis of MRI and NIRF images showed that the binding of Den-RGD to the neovasculature reached a plateau on day 10 after ischemia in both the WT and $d b / d b$ mice. Hence, for the first time, it has been demonstrated that the greatest amount of angiogenesis in ischemic stroke mouse models occurs on day 10. Subsequently, the neovasculature may gradually become mature blood vessels. Increased expression of $\beta_{3}$ integrin was observed and maintained up to 3 weeks after ischemia in the two types of models. Moreover, the signal enhancement observed on MRI and NIRF images was significantly lower in the diabetic mice compared with the WT mice at every time point after ischemia. Diabetes can cause sparse microvessels and diminish collateral circulation [35]. This low-contrast effect might be attributed, in part, to the specific targeting associated with sparse neovascular vessels. Previous study has indicated that the impaired angiogenesis in diabetes is associated with the dysregulation of a complex angiogenesis regulatory network, including vascular endothelial growth factor, placental growth factor, neuropilin-1, and elastin [36]. In combination with the findings in previous studies, it is evident that diabetes diminishes the angiogenic response to cerebral ischemia.

Convincing evidence has suggested that neovascularization is not only caused by endothelial cell proliferation but also correlates with circulating EPCs derived from bone marrow [37]. However, patients with diabetes present an impaired function and decreased number of circulating EPCs [38]. These findings support a potential therapeutic target for patients with diabetes. Most investigations of EPC-based therapy have obtained satisfactory effects in ischemic models of healthy animals [7-9]. Transplanted EPCs are capable of integrating into damaged vasculature and secreting growth factors, cytokines and chemokines to promote angiogenesis. In diabetes, several studies have revealed that vascular damage could only be repaired by healthy but not diabetic EPCs [39]. To stimulate angiogenesis in our cerebral ischemic mouse models, EPC treatment was used. An apparent enhancement of the TBR on the NIRF images after EPC transplantation was observed in both diabetic and WT mice at $24 \mathrm{~h}$ post-injection of Den-RGD. However, the WT mice treated with EPCs exhibited a denser signal intensity enhancement in the peri-infarct area on the $\mathrm{T}_{1}$-weighted images compared with the mice treated with saline, whereas the diabetic mice treated with EPCs exhibited a relatively moderate signal enhancement. The results indicate that the sensitivity of NIRF imaging is higher compared with
MRI. This dual-modal imaging method indicated that the bone marrow-derived EPCs could successfully augment the angiogenic response in diabetic and WT mice, although the augmentation in diabetic mice was not as large as in WT mice. These findings might be explained that partially transplanted cells were functionally impaired in diabetic animals. Previous studies have also suggested that high glucose can decrease the functional competency of EPCs [40]. These data strongly suggest that the dual molecular imaging approach is valuable for monitoring the response to pro-angiogenic therapy in animal models. Although the penetration depth of light is limited, optical imaging will most likely be applied to stroke patients in the near future with the rapid development of intra-arterial catheter imaging technology [41].

\section{Conclusions}

In conclusion, this study demonstrated that the neovasculature-targeted nanoprobe can be used to specifically evaluate angiogenesis in mouse models of ischemic stroke. The non-targeted nanoprobe produced significantly lower enhancement compared with the targeted agent. Molecular imaging demonstrated impaired angiogenesis in diabetic mice and revealed the pro-angiogenic effects of EPC, which is the precursor of endothelial cells. While the current clinical techniques for monitoring the therapeutic efficacy in stroke models, such as the measurement of blood flow, can only detect the large vessels in the later stages of angiogenesis, molecular imaging could be employed to map the early signatures of angiogenesis. In the near future, a non-invasive method similar to the one employed in this work might be valuable for evaluating the effects of pro-angiogenic therapy in patients after ischemic stroke.

\section{Abbreviations}

acLDL: acetylated low-density lipoprotein; CNR: contrast-to-noise ratio; cRGD: cyclic arginine-glycine-aspartic acid; EBM-2: Endothelial Basal Media-2; EPCs: endothelial progenitor cells; ICP-MS: inductively coupled plasma mass spectrometry; mNSS: modified neurological severity score; MRI: Magnetic resonance imaging; MVD: microvessel density; NIRF: near-infrared fluorescence; PBS: phosphate-buffered saline; PET: positron-emission tomography; ROI: region of interest; SPECT: single-photon emission computed tomography; TBR: target-to-background ratio; UEA: ulex europaeus agglutinin; WT: wild-type.

\section{Supplementary Material}

Supplementary Methods, Fig.S1 - Fig.S4.

http://www.thno.org/v04p0787s1.pdf 


\section{Acknowledgements}

This work was supported by the Major State Basic Research Development Program of China (973 Program) (No. 2013CB733802, No. 2010CB933903), the National Nature Science Foundation of China (NSFC, No. 81071125, No. 81371538, No. 81230034) and the Jiangsu Provincial Special Program of Medical Science (BL2013029).

\section{Competing Interests}

The authors have declared that no competing interest exists.

\section{References}

1. Navaratna D, Guo SZ, Hayakawa K, et al. Decreased cerebrovascular brain-derived neurotrophic factor-mediated neuroprotection in the diabetic brain. Diabetes. 2011; 60: 1789-96.

2. Kaarisalo MM, Raiha I, Sivenius J, et al. Diabetes worsens the outcome of acute ischemic stroke. Diabetes Res Clin Pract. 2005; 69: 293-8

3. Li Q, Atochin D, Kashiwagi S, et al. Deficient eNOS phosphorylation is a mechanism for diabetic vascular dysfunction contributing to increased stroke size. Stroke. 2013; 44: 3183-8

4. Zhu M, Bi X, Jia Q, et al. The possible mechanism for impaired angiogenesis after transient focal ischemia in type 2 diabetic GK rats: different expressions of angiostatin and vascular endothelial growth factor. Biomed Pharmacother. 2010; 64: 208-13.

5. Charriaut-Marlangue C, Bonnin P, Gharib A, et al. Inhaled nitric oxide reduces brain damage by collateral recruitment in a neonatal stroke model. Stroke. 2012; 43: 3078-84.

6. Zechariah A, ElAli A, Doeppner TR, et al. Vascular endothelial growth factor promotes pericyte coverage of brain capillaries, improves cerebral blood flow during subsequent focal cerebral ischemia, and preserves the metabolic penumbra. Stroke. 2013; 44: 1690-7.

7. Fan Y, Shen F, Frenzel T, et al. Endothelial progenitor cell transplantation improves long-term stroke outcome in mice. Ann Neurol. 2010; 67: 488-97.

8. Zhang ZG, Zhang L, Jiang Q, et al. Bone marrow-derived endothelial progenitor cells participate in cerebral neovascularization after focal cerebral ischemia in the adult mouse. Circ Res. 2002; 90: 284-8.

9. Taguchi A, Soma T, Tanaka H, et al. Administration of CD34+ cells after stroke enhances neurogenesis via angiogenesis in a mouse model. J Clin Invest. 2004; 114: $330-8$

10. Wang XX, Zhang FR, Shang YP, et al. Transplantation of autologous endothelial progenitor cells may be beneficial in patients with idiopathic pulmonary arterial hypertension: a pilot randomized controlled trial. J Am Coll Cardiol. 2007: 49: 1566-71.

11. Lin W, Xiong L, Han J, et al. External counterpulsation augments blood pressure and cerebral flow velocities in ischemic stroke patients with cerebral intracranial large artery occlusive disease. Stroke. 2012; 43: 3007-11.

12. Winter PM, Caruthers SD, Allen JS, et al. Molecular imaging of angiogenic therapy in peripheral vascular disease with alphanubeta3-integrin-targeted nanoparticles. Magn Reson Med. 2010; 64: 369-76.

13. Schmieder AH, Winter PM, Williams TA, et al. Molecular MR imaging of neovascular progression in the $\mathrm{Vx} 2$ tumor with alphavbeta3-targeted paramagnetic nanoparticles. Radiology. 2013; 268: 470-80.

14. Yan $\mathrm{H}$, Wang L, Wang J, et al. Two-order targeted brain tumor imaging by using an optical/paramagnetic nanoprobe across the blood brain barrier. ACS Nano. 2012; 6: 410-20.

15. Okada Y, Copeland BR, Hamann GF, et al. Integrin alphavbeta3 is expressed in selected microvessels after focal cerebral ischemia. Am J Pathol. 1996; 149: $37-44$

16. Li C, Winnard P Jr, and Bhujwalla ZM. Facile synthesis of 1-(acetic acid)-4,7,10-tris(tert-butoxycarbonylmethyl)-1,4,7,10-tetraaza-cyclododecane: a reactive precursor chelating agent. Tetrahedron Lett. 2009; 50: 2929-31.

17. Watson BD, Dietrich WD, Busto R, et al. Induction of reproducible brain infarction by photochemically initiated thrombosis. Ann Neurol. 1985; 17: 497-504.

18. Chen $\mathrm{R}, \mathrm{Yu} \mathrm{H}$, Jia $\mathrm{ZY}$, et al. Efficient nano iron particle-labeling and noninvasive MR imaging of mouse bone marrow-derived endothelial progenitor cells. Int J Nanomedicine. 2011; 6: 511-9.

19. Klohs J, Grafe M, Graf K, et al. In vivo imaging of the inflammatory receptor CD40 after cerebral ischemia using a fluorescent antibody. Stroke. 2008; 39: 2845-52.

20. Cui X, Chopp M, Zacharek A, et al. The Neurorestorative Benefit of GW3965 Treatment of Stroke in Mice. Stroke. 2013; 44: 153-61.
21. Chen $\mathrm{J}$, Sanberg PR, $\mathrm{Li} \mathrm{Y}$, et al. Intravenous administration of human umbilical cord blood reduces behavioral deficits after stroke in rats. Stroke. 2001; 32: 2682-8.

22. Wang $\mathrm{XY}, \mathrm{Ju} \mathrm{S}, \mathrm{Li} \mathrm{C}$, et al. Non-invasive imaging of endothelial progenitor cells in tumor neovascularization using a novel dual-modality paramagnetic/near-infrared fluorescence probe. PLoS One. 2012; 7: e50575.

23. Xiao Y, Hong $\mathrm{H}$, Matson VZ, et al. Gold Nanorods Conjugated with Doxorubicin and cRGD for Combined Anticancer Drug Delivery and PET Imaging. Theranostics. 2012; 2: 757-68.

24. Krupinski J, Kaluza J, Kumar P, et al. Role of angiogenesis in patients with cerebral ischemic stroke. Stroke. 1994; 25: 1794-8.

25. Sipkins DA, Cheresh DA, Kazemi MR, et al. Detection of tumor angiogenesis in vivo by alphaVbeta3-targeted magnetic resonance imaging. Nat Med. 1998; 4: 623-6.

26. Pan D, Schmieder AH, Wang K, et al. Anti-Angiogenesis Therapy in the Vx2 Rabbit Cancer Model with a Lipase-cleavable Sn 2 Taxane Phospholipid Prodrug using av $\beta 3$-Targeted Theranostic Nanoparticles. Theranostics. 2014; 4: 565-78.

27. Cai W, Chen K, Li ZB, et al. Dual-function probe for PET and near-infrared fluorescence imaging of tumor vasculature. J Nucl Med. 2007; 48: 1862-70.

28. Haubner R, Wester HJ, Weber WA, et al. Noninvasive imaging of alpha(v)beta3 integrin expression using 18F-labeled RGD-containing glycopeptide and positron emission tomography. Cancer Res. 2001; 61:1781-5.

29. Zhu L, Guo N, Li O et al. Dynamic PET and Optical Imaging and Compartment Modeling using a Dual-labeled Cyclic RGD Peptide Probe. Theranostics. 2012; 2: 746-56.

30. Ji $S$, Zheng $Y$, Shao $G$, et al. Integrin $a(v) \beta_{3}$-targeted radiotracer $(99 \mathrm{~m}) \mathrm{Tc}-3 \mathrm{P}-\mathrm{RGD}_{2}$ useful for noninvasive monitoring of breast tumor response to antiangiogenic linifanib therapy but not anti-integrin $\alpha(v) \beta_{3} R_{G} D_{2}$ therapy. Theranostics. 2013; 3: 816-30.

31. Haubner R, Wester HJ, Reuning U, et al. Radiolabeled alpha(v)beta3 integrin antagonists: a new class of tracers for tumor targeting. J Nucl Med. 1999; 40: 1061-71.

32. Choi $\mathrm{H}$, Phi JH, Paeng JC, et al. Imaging of integrin $\alpha(\mathrm{V}) \beta(3)$ expression using (68)Ga-RGD positron emission tomography in pediatric cerebral infarct. Mol Imaging. 2013; 12: 213-7.

33. Hua J, Dobrucki LW, Sadeghi MM, et al. Noninvasive imaging of angiogenesis with a 99mTc-labeled peptide targeted at alphavbeta3 integrin after murine hindlimb ischemia. Circulation. 2005; 111: 3255-60.

34. Sandoval KE, Witt KA. Blood-brain barrier tight junction permeability and ischemic stroke. Neurobiol Dis. 2008; 32: 200-19.

35. Adeghate E. Molecular and cellular basis of the aetiology and management of diabetic cardiomyopathy: a short review. Mol Cell Biochem. 2004; 261: 187-91.

36. Schiekofer S, Galasso G, Sato K, et al. Impaired revascularization in a mouse model of type 2 diabetes is associated with dysregulation of a complex angiogenic-regulatory network. Arterioscler Thromb Vasc Biol. 2005; 25: 1603-9.

37. Asahara T, Murohara T, Sullivan A, et al. Isolation of putative progenitor endothelial cells for angiogenesis. Science. 1997; 275: 964-7.

38. Fadini GP, Miorin M, Facco M, et al. Circulating endothelial progenitor cells are reduced in peripheral vascular complications of type 2 diabetes mellitus. J Am Coll Cardiol. 2005; 45: 1449-57.

39. Caballero S, Sengupta N, Afzal A, et al. Ischemic vascular damage can be repaired by healthy, but not diabetic, endothelial progenitor cells. Diabetes. 2007; 56: 960-7.

40. Chang J, Li Y, Huang Y, et al. Adiponectin prevents diabetic premature senescence of endothelial progenitor cells and promotes endothelial repair by suppressing the p38 MAP kinase/p16INK4A signaling pathway. Diabetes. 2010: 59: 2949-59.

41. Yoo H, Kim JW, Shishkov M, et al. Intra-arterial catheter for simultaneous microstructural and molecular imaging in vivo. Nat Med. 2011; 17: 1680-4. 